\begin{tabular}{|c|l|}
\hline Title & Channeled spectropolarimetry using a coherent white light continuum \\
\hline Author(s) & Somekawa, Toshihiro; Oka, Kazuhiko; Fujita, Masay uki \\
\hline Citation & $\begin{array}{l}\text { Optics Letters, 35(22), 3811-3813 } \\
\text { https:/doi.org/L0.1364/0L.35.003811 }\end{array}$ \\
\hline Issue Date & 2010-11-15 \\
\hline Doc URL & http://hdl.handle.net/2115/47198 \\
\hline Rights & ○ 2010 Optical Society of A merica \\
\hline Type & article \\
\hline File Information & OL 35-22_3811-3813.pdf \\
\hline
\end{tabular}

Instructions for use 


\title{
Channeled spectropolarimetry using a coherent white-light continuum
}

\author{
Toshihiro Somekawa, ${ }^{1, *}$ Kazuhiko Oka, ${ }^{2}$ and Masayuki Fujita ${ }^{1,3}$ \\ ${ }^{1}$ Institute for Laser Technology, 2-6 Yamadaoka, Suita, Osaka 565-0871, Japan \\ ${ }^{2}$ Division of Applied Physics, Faculty of Engineering, Hokkaido University, Sapporo 060-8628, Japan \\ ${ }^{3}$ Institute of Laser Engineering, Osaka University, 2-6 Yamadaoka, Suita, Osaka 565-0871, Japan \\ *Corresponding author: somekawat@ile.osaka-u.ac.jp
}

Received September 1, 2010; revised October 19, 2010; accepted October 20, 2010;

posted October 21, 2010 (Doc. ID 134425); published November 9, 2010

\begin{abstract}
We carry out polarization measurements using a coherent white-light continuum as a light source for channeled spectropolarimetry. The white-light continuum, whose spectrum ranges from the UV to the IR region, is generated in Kr gas by a terawatt femtosecond laser system. The complete set of Stokes parameters from 450-700 nm are reconstructed from one spectral measurement. Also, the effectiveness of channeled spectropolarimetry using a coherent white-light continuum is experimentally demonstrated with a highly attenuating sample whose transmittance is as low as $10^{-6}$. (C) 2010 Optical Society of America
\end{abstract}

OCIS codes: $280.3640,320.6629,260.2130$.

When a high-intensity ultrashort pulse is focused into a transparent medium, a broadband phase-coherent whitelight continuum is generated [1-3]. Recent progress in laser technology, such as chirped pulse amplification (CPA) and a photonic crystal fiber, has made this powerful light source even more versatile in both the physical and life sciences [4-10]. Furthermore, we have demonstrated the use of white-light lasers in lidar systems. This enables nonspherical particles to be monitored through the multiwavelength depolarization ratios expressed as the ratio of $p$ - to $s$-polarization signals $[8,9]$. As a further application for the white-light continuum, here we propose a remote spectroscopic measurement technique based on channeled spectropolarimetry.

Channeled spectropolarimetry is a spectrally broadband method for determining the spectrally resolved polarization state of light from a single spectral measurement $[11,12]$. The method requires no mechanically or electrically controllable polarization components but a continuous spectrum without discrete emission lines. Measurement of a sample's broadband polarimetric signature, in principle, can be accomplished using a conventional tungsten halogen lamp. However, the spectral intensity obtained from the channeled spectropolarimeter using such a lamp is often far too low if high absorption and low scattering coefficients are present within the sample. Highly sensitive channeled spectropolarimetric measurements are therefore of great interest for the weak signals. By combining the properties of channeled spectropolarimetry with the low-divergence properties and broadband nature of a white-light continuum without particular emission lines, the complete set of Stokes parameters for weakly scattered light can be detected over a long distance. The depolarization lidar including the four Stokes measurements may improve classification accuracy of nonspherical particles [13]. Furthermore, remote in situ analysis is also desirable if measurements have to be carried out in hazardous or inaccessible environments.

To establish the principle of using channeled spectropolarimetry with the white-light continuum source, we measure the spectrally resolved polarization state of a linearly polarized beam transmitted by a birefringent sample. The results are in good agreement with theoretical calculations, even when the sample transmittance is as low as $10^{-6}$.

A schematic diagram of the spectroscopic polarimeter with a channeled spectrum, using the coherent whitelight continuum, is given in Fig. 1. A Ti:sapphire laser was used with a regenerative and CPA amplifier to produce $70 \mathrm{~mJ}, 100 \mathrm{fs}$ pulses at a repetition rate of $10 \mathrm{~Hz}$. The center wavelength is $800 \mathrm{~nm}$, and the beam diameter about $4 \mathrm{~cm}$. The femtosecond laser pulses are then focused into the $\mathrm{Kr}$ gas and converted into a white-light continuum. The white-light continuum generated by focusing in a rare gas has a continuous spectrum with high spatial coherence, ranging from the UV to visible to IR range [2]. In addition, the white-light continuum generated in $\overline{\mathrm{K}}$ gas preserves the linear polarization of the fundamental beam so that its polarization axis can be made variable by rotating a half-wave plate within the incident $800 \mathrm{~nm}$ laser beam. In this measurement, the white-light's polarization axis is aligned with the laboratory vertical axis. The light is transmitted by a sample block, consisting of neutral density (ND) filters and a thin quartz plate (TQP) with a thickness of $91.6 \mu \mathrm{m}$. The TQP was originally designed as a zero-order half-wave plate at the wavelength of $1550 \mathrm{~nm}$. A highly absorbing sample was simulated by incorporating ND filters. Light emerging from the sample block then impinges upon a channeled spectropolarimeter. In the spectropolarimeter block, the light

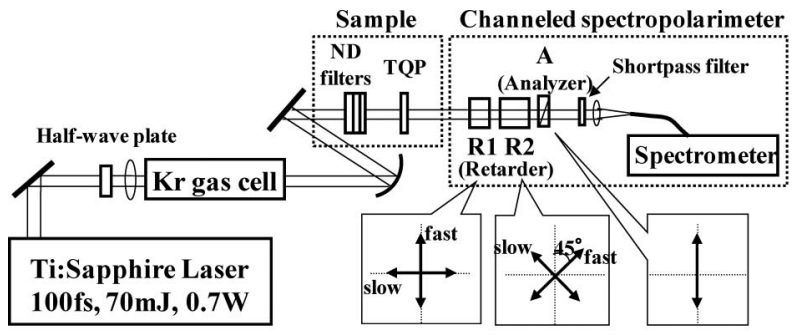

Toshihiro Somekawa

Fig. 1. Schematic of the experimental setup for the spectroscopic polarimeter with a channeled spectrum using the coherent white-light continuum. 
first passes through two high-order retarders $R 1$ and $R 2$, followed by an analyzer $A$. The retarders $R 1$ and $R 2$, chosen for the study, are $z$-cut quartz crystals with thicknesses of 2 and $4 \mathrm{~mm}$, respectively. The axis directions of $R 1, R 2$, and $A$ are fixed such that the fast axes of $R 1$ and $R 2$ are respectively oriented at $0^{\circ}$ and $45^{\circ}$ with respect to the laboratory vertical axis, and $A$ is aligned with the fast axis of $R 1$. The spectrum of the white light, emitted from the analyzer $A$, is recorded using a fiber-coupled spectrometer (BWTEK BRC642E) after suppressing the fundamental $800 \mathrm{~nm}$ laser beam with a shortpass filter that limits our study to the visible region $(450-700 \mathrm{~nm})$ of the white light. The spectral measurements were performed by taking an average of 100 signals to reduce the effects of the white-light spectral fluctuations.

Figure 2(a) shows an example of the obtained channeled spectrum, acquired when the fast axis of the TQP in the sample was oriented at $45^{\circ}$ with respect to the slow axis of $R 1$ and the total transmittance of the ND filters was set to approximately $10^{-4}$. Because the phase retardations of the high-order retarders $R 1$ and $R 2$ are heavily dependent on wavelength, the obtained spectrum is finely modulated; such a spectrum is referred to as a channeled spectrum. The spectrum consists of one low-frequency component and three quasi-cosinusoidal components with different carrier frequencies. Let $S_{0}, S_{1}, S_{2}$, and $S_{3}$ designate the spectrally resolved Stokes parameters [14] of the light emerging from the absorbing sample. The low-frequency component in the channeled spectrum is proportional to $S_{0}$, and the three carrier components carry the information of either $S_{1}$ or $S_{2}+i S_{3}$. Accordingly, the four spectrally resolved Stokes parameters
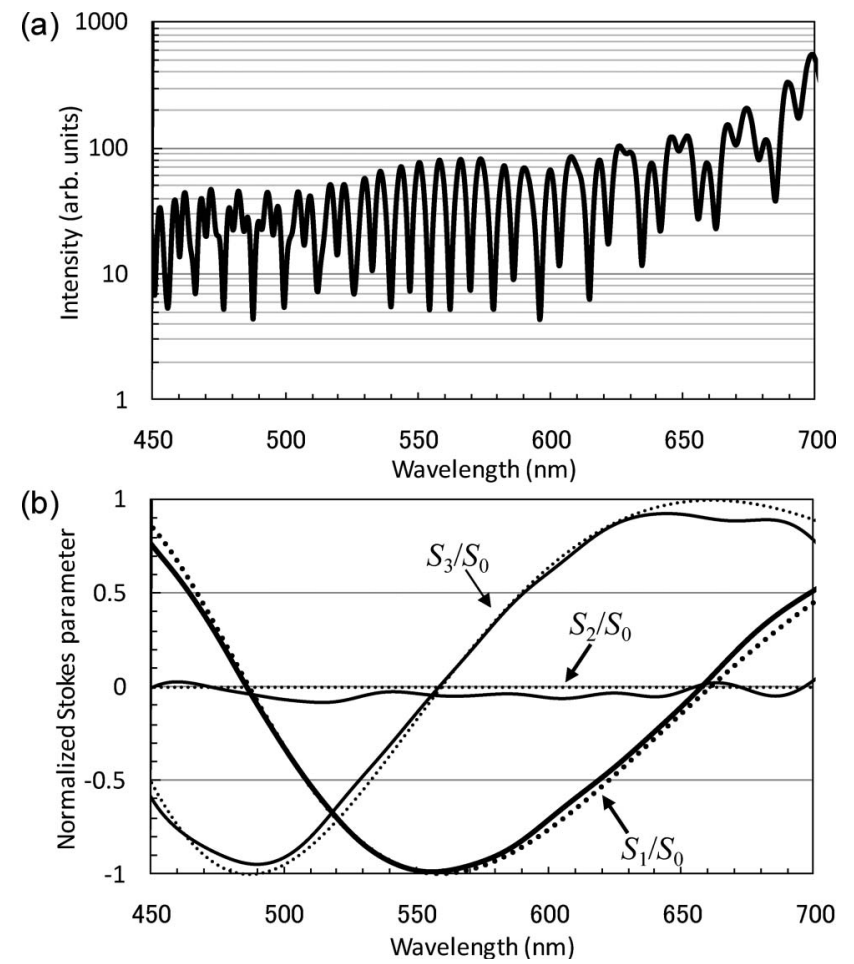

Fig. 2. (a) Channeled spectrum and (b) normalized Stokes parameters for the beam transmitted by the thin quartz plate when the source polarization is oriented at $45^{\circ}$ to the crystal's fast axis. Solid and dotted curves show the experimentally obtained and theoretically calculated values, respectively. can be demodulated simultaneously and independently from the single channeled spectrum by use of Fourier analysis. The normalized Stokes parameters, $S_{1} / S_{0}$, $S_{2} / S_{0}$, and $S_{3} / S_{0}$, after demodulation from the channeled spectrum, are shown in Fig. 2(b) by the solid curves. The standard deviations determined from five sequential spectral measurements, in the same polarization condition, were calculated as $0.003,0.006$, and 0.004 for $S_{1} / S_{0}$, $S_{2} / S_{0}$, and $S_{3} / S_{0}$, respectively. The error bars are smaller than plot symbols and have been omitted for better clarity in this Letter. The theoretically computed normalized Stokes parameters are also shown in Fig. 2(b) by the dotted curves. Using wavelength $\lambda$, the normalized Stokes parameters of the light emerging from the sample block are given by

$$
\left(S_{1} / S_{0}, S_{2} / S_{0}, S_{3} / S_{0}\right)=(\cos \delta(\lambda), 0, \sin \delta(\lambda)),
$$

with $\delta(\lambda)=2 \pi B(\lambda) d / \lambda$ being the phase retardation of the TQP. Here $d$ is its thickness and $B(\lambda)=n_{e}(\lambda)-n_{o}(\lambda)$ is its birefringence, where $n_{e}(\lambda)$ and $n_{o}(\lambda)$ denote the extraordinary and ordinary refractive indices of the crystal, respectively. The theoretical value of the birefringence $B(\lambda)$ of quartz, as a function of wavelength, was computed from the table data of the refractive indices given at discrete wavelengths by Palik [15] using numerical leastsquare fitting. As can be understood from this figure, the experimentally measured and theoretically computed normalized Stokes parameters almost coincide with each other, demonstrating that the channeled spectropolarimeter can be successfully incorporated into the highly absorbing optical system by use of the white-light continuum as a light source. It should be noted that no significant demodulated errors were observed by averaging out the pulse-to-pulse spectral fluctuations of the white-light continuum. In particular, the demonstrated Stokes parameters are free from the errors associated with discrete emission lines, because the spectrum includes no significant emission lines. When another white-light source including emission lines, such as an Xe-lamp, is used with the channeled spectropolarimeter, the obtained spectrum, whose profile is proportional to the source spectrum, also includes the spectral lines. In this case, the three carrier components deviate from the quasicosinusoidal waveforms, and thereby one cannot sufficiently separate the respective components by Fourier analysis. Therefore, the white-light continuum, generated by rare gases, is an appropriate light source for use with channeled spectropolarimetry.

To examine the performance for the remote applications under highly absorbing or scattering conditions, we repeated the acquisition of the channeled spectrum for the TQP by changing the ND filters. Figure 3 shows a typical $S_{1} / S_{0}$ parameter at various transmittances of the sample block. The discrepancy between $10^{-4}$ and $10^{-6}$ transmittances is small: less than 0.1 for the reconstructed $S_{1} / S_{0}$ parameters at $559 \mathrm{~nm}$. This result demonstrates that the present experimental system can be used with a sample whose transmittance is as low as $10^{-6}$. The small discrepancies in Fig. 3 between the data of different sample transmittances can be mainly attributed to background light. With the decrease in the intensity of the light under measurement, the background light level 


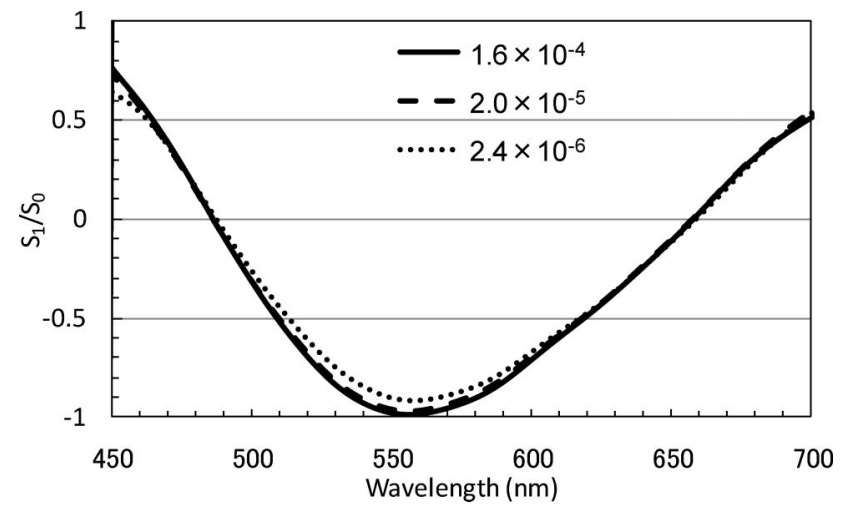

Fig. 3. $S_{1} / S_{0}$ parameter at various transmittances.

becomes relatively apparent. This effect result mainly in the decrease in the absolute values of the measured normalized Stokes parameters, because $S_{1}, S_{2}$, and $S_{3}$, included in the carried components of the spectrum, are almost immune to the background light, whereas $S_{0}$ is demodulated from the low-frequency, namely, noncarried, component.

It should be noted that, in our experimental system, the total transmittance of the ND filters had to be selected to be less than $10^{-4}$ to prevent the saturation of the spectrometer. However, this does not imply that the sample transmittance must be smaller than $10^{-4}$. If we consider the ND filters in Fig. 1 to be included not in the sample but in the illumination optics, the remaining part of the sample, namely the TQP, is almost transparent. Accordingly, our experimental system can be applied with a sample whose transmittance ranges from 1 to $10^{-6}$, provided that the intensity of the white-light continuum can be adaptively controlled by variable ND filters in the illumination optics. This implies that the present system has a potential to measure the complete spectropolarimetric properties of an absorbing or scattering object whose attenuation is up to $60 \mathrm{~dB}$. The wide range for the acceptable sample attenuation and lower detection limit of the white-light continuum makes it a potentially preferred light source for remote channeled spectroscopy. It should be noted that the sensitivity of the present experimental system is not sufficient as it is for use with our lidar system [ㅇ,9] but should be acceptable with other applications in remote polarimetric measurement. We anticipate that we can increase the sensitivity by improving the experimental system, such as by reducing or compensating the effect of the background light and by optimizing the average time, so that the method can be used with the lidar measurement.

This study was supported by a Grant-in-Aid for Scientific Research (20519004) from the Ministry of Education, Science, Sports, and Culture of Japan (MESSC-JP).

\section{References}

1. J. M. Dudley, G. Genty, and S. Coen, Rev. Mod. Phys. 78, 1135 (2006).

2. H. Nishioka, W. Odajima, K. Ueda, and H. Takuma, Opt. Lett. 20, 2505 (1995).

3. J. Kasparian, R. Sauerbrey, D. Mondelain, S. Niedermeier, J. Yu, J.-P. Wolf, Y.-B. Andre, M. Franco, B. Prade, S. Tzortzakis, A. Mysyrowicz, M. Rodriguez, H. Wille, and L. Woste, Opt. Lett. 25, 1397 (2000).

4. P. Li, K. Shi, and Z. Liu, Opt. Lett. 30, 156 (2005).

5. S. A. Trushin, W. Fuss, K. Kosma, and W. E. Schmid, Appl. Phys. B 85, 1 (2006).

6. H. Kano and H. Hamaguchi, Appl. Phys. Lett. 86, 121113 (2005).

7. J. Kasparian, M. Rodriguez, G. Mejean, J. Yu, E. Salmon, H. Wille, R. Bourayou, S. Frey, Y.-B. Andre, A. Mysyrowicz, R. Sauerbrey, J.-P. Wolf, and L. Woste, Science 301, 61 (2003).

8. T. Somekawa, C. Yamanaka, M. Fujita, and M. C. Galvez, J. Appl. Phys. 103, 043101 (2008).

9. T. Somekawa, C. Yamanaka, M. Fujita, and M. C. Galvez, Jpn. J. Appl. Phys. 47, 2155 (2008).

10. T. Somekawa, M. Fujita, and Y. Izawa, Appl. Phys. Express 3, 082401 (2010).

11. K. Oka and T. Kato, Opt. Lett. 24, 1475 (1999).

12. H. Okabe, M. Hayakawa, H. Naito, A. Taniguchi, and K. Oka, Opt. Express 15, 3093 (2007).

13. J. D. Houston and A. I. Carswell, Appl. Opt. 17, 614 (1978).

14. R. M. A. Azzam and N. M. Bashara, Ellipsometry and Polarized Light (North-Holland, 1988).

15. E. D. Palik, Handbook of Optical Constants of Solids I (Academic, 1985). 